\title{
El dolo eventual y la culpa consciente en los accidentes automovilísticos: la perspectiva desde el derecho penal argentino
}

\section{Fraud and conscious guilt in car accidents}

DOI: http://dx.doi.org/10.17981/juridcuc.13.1.2017.10

Artículo de investigación. Fecha de recepción: 21/06/2017 Fecha de aceptación: 11/09/2017

\author{
Christian Andrés Pérez Sasso ${ }^{1}$ \\ Universidad de Buenos Aires (Argentina) \\ cs@estudiorys.com.ar
}

Para citar este artículo:

Pérez, C. (2017). El dolo eventual y la culpa consciente en los accidentes automovilísticos: la perspectiva desde el derecho penal argentino. JURÍDICAS CUC, vol. 13, no. 1, pp. 213-232. DOI: http://dx.doi.org/10.17981/juridcuc.13.1.2017.10

\section{Resumen}

La doctrina y jurisprudencia no es pacífica en situar parámetros claros de separación entre el dolo eventual y la culpa consciente. Esto conlleva, en países como Argentina, en donde los siniestros vehiculares es uno de los índices de mortalidad más alta, la dificultad en determinar el grado de responsabilidad penal. A continuación, se analizará la repuesta que ofrece el derecho argentino, y así evaluar si la solución legislativa se condice con los postulados y principios dogmáticos.

\section{Palabras claves}

Dolo eventual, culpa consciente, culpa con representación, siniestros vehiculares

\footnotetext{
${ }^{1}$ Abogado de la Universidad de Buenos Aires, matriculado en la Ciudad Autónoma de Buenos Aires; socio en el estudio jurídico Ramos \& Sasso; ayudante de segunda en la asignatura Practico Profesional (Comisión 1090); Disertante en el "Congreso Penal: política criminal y estado de derecho" Comisión III (Dogmática Penal), trabajo "El Dolo Eventual y la Culpa Consciente en los accidentes automovilísticos"; Disertante en "Programa de formación de Práctica profesional", Taller de derecho procesal penal, el 19 de mayo de 2016, organizado por la agrupación universitaria Franja Morada de la Facultad de Derecho U.B.A., Disertante en la jornada académica "Dos años de vigencia de la ley 1761 de 2015 (Rosa Elvira Cely)", el 6 de julio de 2017, organizado por la Universidad de La Costa (Barranquilla), investigador en el proyecto UBACyT "La ignorancia deliberada en los delitos económicos, tributarios y contra la administración pública”.
}

- The author; licensee Universidad de la Costa - CUC. JURÍDICAS CUC vol. 13 no. 1, pp. 213-232. Enero - Diciembre, 2017 Barranquilla. ISSN 1692-3030 Impreso, ISSN 2389-7716 Online 


\section{Abstract}

Legal doctrine and case law doctrine about the difference between fraud and conscious guilt are not undisputed. In some situations, like traffic accidents, the difference is difficult to establish. This paper will focus on the analysis of this theme and try to give an answer from the contemporary criminal theory and, thus, study if there is a solution to this current problem and examine if these conducts are intentional or negligent types.

\section{Keywords}

Dolus eventualis, Conscious guilt, Represented guilt, road traffic accidents

\section{INTRODUCCIÓN}

La dogmática penal determina que una conducta es punible cuando reúne una serie de requisitos: una acción o conducta típica, antijurídica y culpable (Stratenwerth, 2016).

Durante más de sesenta años se ha discutido la ubicación del dolo. Para el casualismo se encuentra en la culpabilidad y para el finalismo en la tipicidad. Esta discusión en la actualidad se encuentra superada, siendo aceptada la teoría finalista de la acción elaborada por Welzel: todo accionar voluntario tiene una finalidad, por lo que el sujeto activo se representa mentalmente un fin buscado, selecciona los medios y pone en funcionamiento ejecutando su cometido en la dirección de la finalidad representada (Zaffaroni, Alagia y Slokar, 2007); en cambio, en los tipos culposos, la finalidad de la acción se encuentra en la mala selección de los medios.

Esta concepción explica más fidedignamente el plano fáctico, ya que el dolo (conocimiento y voluntad) se caracteriza por estar situado en la faz interna del sujeto (Pérez, 2011), que traerá consecuencia en su aspecto externo; por esta razón, el dolo debe situarse en la representación y voluntad (Stratenwerth, 2016). De esta manera, el correcto lugar del dolo es la tipicidad, debido a que debe analizarse en el tipo si lo acontecido en el mundo 
fue consecuencia de un querer interno y dejar en la culpabilidad la conciencia de la antijuridicidad (Tenca, 2010).

Los siniestros vehiculares son una de las causas de muertes con mayor preponderancia, siendo necesario evaluar cuál es la repuesta que otorga la dogmática penal y la legislación y jurisprudencia argentina. La cuestión a averiguar es, en estos tipos de casos, cómo la delgada diferencia entre el dolo eventual y la culpa consciente o con representación se manifiesta. Quien ocasiona lesiones o la muerte tras pasar un semáforo en rojo, ¿actúa con dolo o culpa? ¿Cambia el análisis en el caso de que el autor del delito esté desarrollando una competencia callejera ilegal? ¿En casos como estos cómo se ha pronunciado la jurisprudencia? ¿Qué ventajas o desventajas otorga el derecho penal argentino?

\section{Estructura del delito doloso y los niveles de dolos}

El tipo penal, en su estructura, está compuesto por dos elementos: el objetivo y el subjetivo. Tras el análisis del primero (sujeto activo del delito, la acción típica, resultado típico, si se requiere un resultado o meramente un peligro, los elementos descriptivos y normativos), se debe determinar si el segundo se encuentra presente: el dolo.

El dolo consiste en el conocimiento y voluntad realizadora de los elementos del tipo objetivo. En palabras de Zaffaroni et al. (2007): "El dolo es definido como la voluntad realizadora del tipo guiada por el conocimiento de los elementos objetivos del tipo, siendo este último siempre efectivo" (p. 403).

A su vez, pueden aparecer especiales elementos subjetivos del tipo: determinada intención, motivación, estado de ánimo o enfatizar lo que se sabe (Garibaldi y Pitlevnik, 2007). Así, se observa que el dolo es un límite que marca mayor pena para el delito que se trate desde la perspectiva que el dolo pone en evidencia una voluntad que busca realizar el resultado típico, siendo el límite de la imputación (Terragni, 2009). En este marco, el dolo debe existir al tiempo del comienzo de ejecución, pudiendo fraccionarse el dolo cuando la realización misma tiene actos plurales (Garibaldi y Pitlevnik, 2007). 
Existen tres niveles de dolo (Bacigalupo, 1999): el dolo de intención o propósito (es lo que el sujeto activo persigue), el dolo directo (son aquellas consecuencias que, si bien no se buscan, ocurrirán con seguridad) y el dolo eventual (quien no persigue un resultado ni lo prevé como seguro, pero que igualmente continua su accionar y asume el resultado voluntariamente).

Dolo de intención

En un primer momento hay que tener claro que el dolo se fragmenta en dos partes: aspecto cognoscitivo y aspecto volitivo. De este modo, el plan criminal (intercriminis) se puede definir de la siguiente manera: El sujeto activo se propone un fin, selecciona los medios para su persecución, en consecuencia, se retrotrae mentalmente al momento de proposición de dicho fin y pone en marcha la causalidad según los medios elegidos para llegar a la finalidad propuesta. (Argibay, Damianovich, Moras y Vergara, 1972).

En este nivel, basta que el conocimiento preste la posibilidad, aunque sea escasa, de obtener el resultado. Es conteste la doctrina en exigir que objetivamente el sujeto activo se represente la posibilidad de alcanzar la conducta tipificada por la norma, asimismo, Roxin (2007) sostiene: "Los resultados deseados y causados poseen intención, a pesar que su producción no sea seguro o sea el fin último a alcanzar" (p. 419).

La intención puede manifestarse de dos maneras, o bien cuando se requiere un daño más allá del comportamiento ejecutado, o cuando el tipo se concreta con la lesión a un bien jurídico determinado.

Es crucial dejar en claro que la intención está presente, aunque el resultado no ocurra como se espera; como defiende Sancinetti (1991) se puede disponer de dudas para llegar al resultado propuesto o la consecución del fin para el autor puede ser incierta (p. 146-147).

\section{Dolo directo}

El dolo de primer grado abarca también el segundo grado, pero este último no al primero. En este sentido, el dolo de "conse- 
cuencias necesarias" se presenta cuando hay una concreta seguridad en el resultado y ésta es asumida por el realizador de la acción (el ejemplo clásico es el terrorista que con el fin de asesinar al embajador coloca una bomba en el auto diplomático, dando por sentado que además del político le dará muerte al conductor del vehículo). Así ha resuelto en numerosas ocasiones la jurisprudencia alemana diciendo que las consecuencias de la acción que se reconocen como necesarias son asumidas en su voluntad por el agente, aun cuando no tenga en absoluto interés en esas consecuencias.

\section{Dolo eventual}

Es prudente analizar previamente las distintas teorías que intentan explicar el dolo de tercer grado. Los esfuerzos doctrinarios se dirigieron hacia dos direcciones: cuál es el límite del dolo y de qué modo se fundamenta su aparición en la modalidad eventual. A continuación, se citarán las distintas teorías que se han elaborado con la finalidad de explicar el dolo eventual.

Teoría del consentimiento. Teniendo como base que el dolo se configura con dos aspectos (cognoscitivo y volitivo), entre el resultado y el accionar debe haber una relación de voluntad; en palabras de Tenca (2010): "El autor debe declararse conforme con la actualización del resultado" (p. 36). Esta tesis es criticada por Roxin, ya que considera que la aprobación del resultado es dirimente para determinar el monto de la pena, pero no influye en el carácter doloso de la conducta; el elemento volitivo se completa con que el autor incluya en su plan criminal el resultado y lo haya querido.

La teoría positiva del consentimiento. El despliegue doloso se presenta cuando hay una resignación al resultado a pesar de que no lo quiera o desee.

La teoría de la representación. Sostiene que la representación de la posibilidad debería ser un motivo contundente para dejar de actuar, y que la confianza en la no producción del acto típico reúne la negación de la posibilidad de que ocurra. Así, los autores alemanes dicen que en esta concepción se percibe 
la posibilidad abstracta de producción del hecho típico, pero negaría en su consciencia la posibilidad de que ocurra el resultado.

La teoría de la indiferencia. Internamente, el sujeto aprueba (o es indiferente) a las posibles consecuencias negativas actuando con dolo, en cambio, cuando dichas consecuencias las evalúa como indeseables, su comportamiento seria culposo. Esta doctrina no hace una lectura satisfactoria, ya que lo transcendental debe ser la decisión y no los deseos.

Las teorías ecléticas. Son una combinación de la teoría de representación y la del conocimiento. En el aspecto cognoscitivo se requiere conocer la peligrosidad de la conducta y en el volitivo que uno se resigne, acepte, se conforme o lo asuma.

La teoría de la probabilidad. El requisito del dolo sería que la probabilidad sea más que la mera posibilidad, pero menor que la probabilidad predominante.

La teoría de la evitabilidad del resultado. Cuando el sujeto se ha representado la posibilidad del resultado, pretende negar el dolo eventual solo cuando la voluntad conductora del sujeto estuviera dirigida a la evitación del resultado.

Teoría de Jakobs. Hay dolo eventual cuando el sujeto evalúa la comisión del tipo como producto de su accionar no siendo improbable su producción.

Teoría de Roxin. Existe certeza de la realización de la conducta típica y, sin embargo, continua con su accionar, resignándose (aceptando) a la eventual realización del delito.

El dolo eventual surgió desde la política criminal para dar repuesta a ciertas conductas a la que la penalidad del delito culposo quedaba disminuida con base en la conducta desplegada, haciendo imposible cumplir la prevención general positiva de la pena, mostrándose como una solución más justa. A su vez, la falta de unanimidad con respecto al concepto de dolo eventual imposibilita, por parte de la jurisprudencia en general, aplicarlo pacíficamente: la existencia de diferentes teorías no permite poseer una sola que sea aceptada por la comunidad jurídica y, a su vez, todas las teorías, como se observa, poseen aporías (Pérez, 2011). 


\section{Estructura del delito culposo}

La estructura culposa se caracteriza por que la dirección de la voluntad no va dirigida a un fin ilícito, sino que, con el objetivo de obtener una finalidad lícita lo ejecuta con una conducta imprudente o negligente (Rusconi y Kierszenbaum, 2016). Es decir, se llega a un comportamiento prohibido por una mala selección de los medios y no por un querer voluntario.

El delito imprudente consiste en la violación de una norma de cuidado, presentando en su génesis una estructura objetiva y una subjetiva (Cesano y Comuñez, 2015).

En la primera se requiere una serie de condiciones: sin excepción, arribar a un resultado típico; el agente debe obrar con una violación del deber de cuidado (debiendo ser analizada en cada caso concreto por lo que no existe una violación del deber de cuidado de índole genérica, asimismo, este principio da origen al "principio de confianza", según el cual una persona confía que otra debe comportarse de acuerdo con el deber de cuidado, siempre y cuando no haya motivos para considerar lo contrario); y tiene que existir un nexo de determinación (conocida como imputación objetiva en el tipo culposo; la violación del deber de cuidado tiene que ser la causa determinante del resultado dañoso).

En el tipo subjetivo, la respectiva violación del deber de cuidado debe haber sido querido por el autor, pudiendo ser con conocimiento del peligro (culpa consciente) o sin conocimiento (culpa inconsciente).

En la culpa con representación, si bien se conoce el riesgo de la conducta, se confía en que el resultado típico no sucederá. En la culpa sin representación, a pesar de poseer los medios para representarse el peligro de su accionar no lo hace.

Como describe Roxín (2007), el tipo subjetivo de la imprudencia consciente reúne la representación de todas las circunstancias del hecho como un peligro no permitido y en la confianza de la no realización típica; por otro lado, en la inconsciente, en cambio, falta el tipo subjetivo, ya que no existe la representación de los presupuestos objetivos (p. 1022). 


\section{El fenómeno de los accidentes de tránsito}

El vertiginoso sistema capitalista condujo la realización de un sin número de actividades que conllevan un riesgo para la sociedad y el manejo vehicular no fue la excepción. Asimismo, la conjunción de una serie de factores, como la falta de infraestructura de autopistas o carreteras, la falta de educación vial, la excesiva cantidad de automóviles de transporte en la metrópolis y la violación de las normas de tránsito ha provocado consecuencias funestas, tanto en la Argentina como en el extranjero.

Las políticas de prevención implantadas en la Argentina no brindaron los resultados esperados, siendo que por medio de una política legislativa inflacionaria se intentó persuadir aquellas conductas que podían provocar consecuencias funestas en la conducción vial.

A esto se sumó el componente social mediante la presión ejercida por la opinión pública y los medios masivos de comunicación, produciendo un sinnúmero de intereses en pugna a los que la jurisprudencia le intento dar una repuesta.

El dolo eventual vs la culpa consciente en los accidentes de tránsito

Llegado a este punto es pertinente analizar qué figura debe aplicarse en los siniestros vehiculares. Previo a examinar la aplicación de una tipología u otra, es posible limitar el campo de casos que se están analizando: nos circunscribimos a aquellos supuestos en los que el exceso de velocidad, adelantarse cuando el semáforo habilitante no lo permite, conducir bajo los efectos del alcohol o los estupefacientes, o el desapego de las normas de tránsito ocasionan lesiones o la muerte.

A continuación, se citarán varios fallos de la jurisprudencia argentina para evaluar qué criterios fueron seguidos por los jueces para configurar en cada hipótesis el delito doloso en grado eventual o el culposo con representación. 


\section{Culpa consciente}

1. CCrim $1^{\circ}$ nominación Catamarca (2007). Cabe condenar por homicidio culposo a quien conduciendo bajo los efectos de estupefacientes a una velocidad excesiva, embiste a un peatón provocándole la muerte. La capacidad de previsión se vio disminuida por el efecto del narcótico, por lo que no pudo representarse el resultado de su accionar.

2. CPenal Jujuy, Sala I (2007). En esta resolución se analizó el siguiente supuesto de hecho: manejar en contra mano, en estado de ebriedad, a excesiva velocidad y ocasionar un resultado dañoso (muerte y lesiones). La Cámara sostuvo que el comportamiento fue a título de culpa basándose en el siguiente razonamiento: "El tránsito a contramano es una imprudencia de alto grado, un quebramiento de las normas de cuidado..., pero no constituye una evidencia de la voluntad del sujeto de dañarse a sí mismo o a otros...".

3. TRcrim $n^{\circ} 6$ Morón (2006). Debe por no tenerse acreditado el dolo eventual al no haber elementos probatorios que pongan en evidencia la representación de la muerte y las lesiones, como así también, actuar con desprecio de esa posibilidad. Todo esto más allá que el sujeto actuó bajo los efectos del alcohol y sin los anteojos que debía utilizar para poder manejar.

4. Cam. Nac. Casación Penal Salla III (2005). La sola circunstancia que el imputado maneje a alta velocidad no es condición reflejante del dolo eventual, debido a que se debe demostrar la consciencia del riesgo, que el mismo fue asumido y no hubo una verdadera renuncia a la evitación del resultado.

5. Cam. Nac. Del Crim y Corr., Sala II (1977). El taxista, con conocimiento del mal estado de los neumáticos, la falta de frenos y conducir a excesiva velocidad comete solamente un delito culposo, ya que estas circunstancias le permitieron prever un accidente que no fue querido por el autor, por lo que no se configura la figura del dolo eventual. 


\section{Dolo eventual.}

1. CRim $N^{\circ} 1$ Trelew (2002). El conductor de un camión de grandes dimensiones, que se dirigía a contramano y con una dosis alta de alcohol en sangre generó la muerte de otro automovilista. La Cámara sostuvo que concurrió homicidio simple con dolo eventual porque el conductor al tener veinte años de experiencia debió representarse como posible el resultado de muerte.

2. CGaranPen Quilmes, Sala I (2001). Existe dolo eventual cuando el encartado conduce sin luces, a alta velocidad (sabiendo que en esa zona pasaban peatones que no podían usar la calzada), con lluvia, sin el respectivo funcionamiento del limpiaparabrisas, y que tras atropellar a cuatro personas llevó a una enganchada del cabello en su auto. Se consideró que el autor sabía que su cometido podía ocasionar el resultado acaecido, despreciando a su vez la vida humana.

3. Capel Noroeste Chubut, Sala B (1998). Un camión, previo a cruzarse al carril contrario, colisionó con un auto que iba en una dirección contraria provocando lesiones a cuatro personas y la muerte de otras diez. Se comprueba que la acción desplegada se ejecutó con total desidia, pudiendo representarse el resultado típico en todo momento y a pesar de ello no desistió en su comportamiento.

4. $3^{\circ}$ CrimCOrr, La Plata, Sala II (1995). No es posible encuadrar en el delito culposo la acción del conductor que viviendo en Argentina conoce la problemática sobre los accidentes de tránsito. El estado de ebriedad provocado no evitó disponer su conciencia de antijuridicidad, no disponía de registro de conducir teniendo conocimiento que le estaba imposibilitado manejar sin la documentación respectiva y el estado de alcoholismo fue voluntariamente provocado.

5. Cpen Santa Fe, Sala I (1986). El imputado que embiste a una persona en un lugar oscuro, con nulo tráfico, comete dolo, aunque sea en grado eventual, aun cuando presume que puede estar en grave peligro. 
El universo de fallos citados precedentemente pone en evidencia el criterio dispar con que los jueces argentinos han resuelto en análogas situaciones. Ante supuestos de hechos muy parecidos (sino iguales) se han tomado determinaciones muy diferentes, ocasionado una suerte de inseguridad jurídica al no haber un criterio uniforme de decisión.

\section{El emblemático caso Cabello}

El siguiente es un caso que tuvo que decidir la justicia argentina en el año 1999 (República de Argentina. Cám. Nac. de Casación Penal Sala III, 2005), el cual tuvo una notoria resonancia en los medios masivos de comunicación. La condena social fue un elemento valorado por los magistrados intervinientes, quienes, como se verá a continuación, discreparon de instancia a instancia en lo referente al dolo eventual y la culpa consciente a la hora de fallar.

Un joven que al volante de un vehículo "preparado" corriendo una picada por la Avenida Cantilo (Ciudad Autónoma de Buenos Aires) junto con otro inmovilista, chocó a un vehículo desde atrás, lo que género que este último se incendiara y murieran sus dos tripulantes. En un primer momento, el tribunal oral consideró que se trataba de un doble homicidio simple con dolo eventual. La Cámara de Casación Penal cambió el criterio calificándolo como doble homicidio cometido en su faz culposa; asimismo, tras este pronunciamiento judicial se modificó el artículo 84 del Código Penal de la Nación (elevando a dos años el mínimo de seis meses cuando el hecho sea en circunstancia de tráfico vehicular).

El Tribunal Oral fundó el dolo eventual en la teoría de la indiferencia (la actitud interna del autor aprobando interiormente el resultado o mostrándose indiferente ante el mismo). Se consideró que estaba probado que Sebastián Cabello decidió, sin motivos de apuro y con aceptación del riesgo por ambos, correr una picada ilegal por la Avenida Cantilo a velocidad antirreglamentaria realizando una abrupta maniobra hacia la derecha y embistiendo por atrás al Renault 6 en el que circulaban Celia E. González Carman y su hija Vanina Rosales, 
provocando la muerte por carbonización. Sustentándose en la educación, sapiencia, volición y lucidez, Cabello actualizó su conocimiento representándose estos resultados finales como posibles y continuos en esa conducta de correr, siéndole indiferente la vida humana.

El tribunal manifestó que se cumplen los aspectos objetivos y subjetivos del delito típico. El hecho en cuestión determinó que se encontraba probado, ya que Cabello puso su energía apretando fuerte el pedal al conducir velozmente el Honda Civic, teniendo solo facultades de dominio interruptivas de su acelerada acción, demostrando que su voluntad no fue la de frenar el comportamiento desplegado (Vitale, 2013). De esta manera, el Tribunal fundó que el Honda Civic funcionó como un arma letal en el resultado obtenido al superar lo preceptuado por la ley; con la aceptación en la participación de una carrera despreocupándose del suceso eventual que estaba pasando. Existía entonces una decisión volitiva y cognoscitiva, con conciencia de peligro, al igual de que internamente hubo consentido la mera posibilidad productiva de los resultados dañosos.

La Cámara de Casación Penal cambió el criterio al considerar que hay una defectuosa fundamentación y elección del encuadre jurídico. Así, sostienen que la circunstancia de conducir a alta velocidad violando el deber de cuidado, confiando en su habilidad como conductor no es factor determinante para la concurrencia del dolo eventual.

El tribunal expresó que se debe demostrar que el autor fue consciente del peligro creado, asumiéndolo y que no tuvo una verdadera renuncia en la evitación del resultado, extremos que por cierto no habían sido probados.

Defiende Casación que fundamentar la representación del resultado muerte basándose en cualidades personales del imputado no son útiles para fundamentar el tipo escogido por el Tribual Oral. En esta inteligencia sostienen que no habría que descartar la confianza de evitación del imputado cuando no ha sido probado en autos que haya visto al auto de la víctima y, además, está acreditado que intentó frenar antes de chocar. No se indica con elementos probatorios que Cabello, al conducir como lo hizo previamente, conoció y aceptó embestir a otro rodado. 
Finalmente, se manifiesta que el imputado actuó con un alto nivel de imprudencia, con desapego de las normas que debía cumplir, pero descartando que haya habido una intención de dañarse a él mismo o a los terceros. Critica el fallo del Tribunal en la repetición de los mismos conceptos, intentando una suerte de convencimientos, y consideran que equiparar el auto a un arma (por su preparación) no establece de por sí que el delito sea intencional, pudiendo un arma propia utilizarse para cometer delitos dolosos o culposos. Asimismo, acentúan que no hay pruebas suficientes que muestren que Cabello efectivamente se representó el resultado muerte. Se desvalorizó el hecho del "tunning" en el rodado, elementos innecesarios para hacer al vehículo con más potencia automovilística. En conclusión, para el Tribunal de Casación Penal, Cabello cometió un doble homicidio culposo.

\section{La ley 23.347 de 2017}

El seis de enero de 2017 se sancionó legislativamente en Argentina la ley 27.347. Ésta incorporó al Código Penal de la Nación el artículo 84 bis consagrando el homicidio bajo la modalidad de "culpa temaría" cuando se da alguna de las siguientes circunstancias:

El conductor se diere a la fuga o no intente socorrer a la víctima siempre y cuando no incurriere en la conducta prevista en el artículo 106, o estuviese bajo los efectos de estupefacientes o con un nivel de alcoholemia igual o superior a quinientos (500) miligramos por litro de sangre en el caso de conductores de transporte público o un (1) gramo por litro de sangre en los demás casos, o estuviese conduciendo en exceso de velocidad de más de treinta (30) kilómetros por encima de la máxima permitida en el lugar del hecho, o si condujese estando inhabilitado para hacerlo por autoridad competente, o violare la señalización del semáforo o las señales de tránsito que indican el sentido de circulación vehicular o cuando se dieren las circunstancias previstas en el art. 193 bis, o con culpa temeraria, o cuando fueren más de una las víctimas fatales. (República Argentina, Congreso de la Nación Argentia, Ley 23.347, 2017) 
De este modo, el poder legislativo con la fuerza de ley sancionó una figura dogmática que no tenía recepción en el código penal argentino ni en sus leyes especiales: la culpa temeraria. La solución propuesta por parte del congreso nacional marca una política criminal que busca persuadir el incumplimiento grave de las normas de tránsito.

Si bien se debe ser críticos con ciertos indicios descriptos en el tipo penal (el análisis de una situación posterior al hecho -huir de la escena sin socorrer a la víctima- no es un elemento que pueda medir la realización dolosa o culposa de una situación exante), el legislador recurrió a una figura penal que aumenta los mínimos y máximos del delito imprudente con representación, permitiendo al juzgador tener una amplia brecha de aplicación de pena, la cual tendrá un quantum mayor si la conducta es temeraria (grave imprudencia). En esta línea, se obtiene un resultado más justo sin tener que desdoblar esfuerzos para encuadrar el accionar bajo la figura de dolo eventual, siendo de especial utilización en aquellos casos en el que el aspecto subjetivo de la tipicidad no presenta una clara demostración de la voluntad realizadora del tipo.

\section{Propuesta teórica y práctica}

Las lesiones o muertes en ocasión de siniestros automovilísticos con base en la legislación penal argentina se producen con una estructura culposa con representación basadas en la conceptualización del dolo de peligro y de lesión, y a la finalidad de los tipos penales.

Se describió en la primera parte la configuración dolosa (en sus tres grados) y culposa de los delitos en general. Posteriormente, se hizo hincapié en cómo la jurisprudencia fue resolviendo la problemática en los accidentes viales. Es por ello que, de acuerdo con lo expuesto y al análisis jurídico elaborado se desarrollará una teoría que posee las siguientes hipótesis: 
1. Las muertes o lesiones sucedidas en un siniestro automovilístico como producto de una violación del deber de cuidado (sea grave o no) se produce con culpa con representación. Entran en ese universo de casos: maniobras que superan el riesgo permitido, avanzar sin el semáforo habilitante o la realización de carreras callejeras.

2. Si el obrar negligente es superador de la violación del deber de cuidado, el dolo eventual de este autor es de peligro y no de lesión en los accidentes automovilísticos graves.

3. El dolo de peligro no se presenta en los tipos de lesión, ya que estos requieren una finalidad. Por ende, a pesar del esfuerzo del intérprete de adecuar estas conductas a la tipicidad dolosa de muerte o lesiones (en su grado eventual), hay que considerarlas como una grave violación del cuidado.

4. El homicidio y las lesiones dolosas son tipos de lesión, por lo que no es subsumible la acción de alguien que ocasiona estas consecuencias por medio de un siniestro automovilístico, siéndoles aplicable la tipología culposa.

En primer lugar, el marco de la presente teoría tiene sus cimientos en la diferencia entre el dolo de peligro y dolo de lesión elaborada por la autora española Teresa Rodríguez Montañes en su obra Delitos de peligro, Dolo e imprudencia (2004). Para poder evaluar qué tipo de dolo puede acaecer en un accidente automovilístico es pertinente analizar su estructura en general.

Sin excepción, el resultado siempre debe ser consecuencia del riesgo creado y no se requiere que el autor tenga pleno conocimiento de todas las circunstancias que ocasionan la muerte o las lesiones. Es decir, de aquella representación del riesgo o peligro sumando la confianza en la no producción del resultado lesivo, ocasiona que el sujeto activo dé por sentado el resultado típico. Es así que el dolo de peligro y el de lesión no son idénticos, siendo el segundo abarcado por el primero, pero no al contrario. De esta manera, se observa que el dolo de peligro es previo al de lesión, siendo preciso para su existencia la situación de riesgo y no el resultado lesivo. 
En ambas modalidades de dolo (peligro y lesión) es pertinente la posibilidad de peligro concreto: consintiendo o resignándose (dolo eventual de lesión y dolo directo de peligro), o bien que no se conforme confiando en tener dominio de la situación y control del peligro evitando la lesión (no hay dolo de lesión pero si de peligro).

Los siniestros automovilísticos de gravedad están situados en la segunda especie, ya que existe una conciencia de peligro concreto pero se confía en tener la capacidad de evitar la lesión (si no aceptaría su autolesión), pero a medida que acepta cierto nivel de riesgo no tiene más remedio que conformarse con la puesta en peligro propia y de terceros; el automovilista que actúa en este marco de acción asume conscientemente la seguridad de resguardar el bien jurídico protegido afirmando el dolo de peligro, pero confiando en dominar el curso causal evitando el respectivo daño, negando el dolo eventual de lesión. Según lo expuesto, la diferencia se encuentra en el elemento volitivo.

En esta inteligencia, Teresa Rodríguez Montañes (2004) enseña que el dolo de peligro presenta dos elementos: uno cognitivo (consciencia de peligrosidad) y un elemento volitivo (voluntad de realizar el peligro). En el primero, se deben reunir todos los elementos objetivos del tipo y se necesita que la representación sea coincidente con la realización, como así también, dicha actualización interna se debe referir al bien jurídico protegido que dependerá de cada tipo penal (el juicio de peligro es asertivo: valora la producción como segura cayendo en dolo directo, o creación como posible adecuándose al dolo eventual). Por su parte, el componente volitivo está configurado por la representación del peligro en el que el sujeto acepta o se conforma con la puesta en peligro.

El comportamiento de un sujeto activo que provoca una muerte o lesión en ocasión de un accidente automovilístico si lo realiza con dolo sería de peligro y no con dolo de lesión. En este punto es pertinente realizar la siguiente pregunta: ¿los tipos penales de homicidio o de lesiones dolosas son tipos de peligro o de lesión?

En los tipos de lesión, según Roxin (2007) se requiere que la acción típica alcance una determinada donosidad de los bienes 
jurídicos protegidos, cumpliendo siempre los presupuestos de la imputación objetiva: el autor debe crear un riesgo no abarcado por el riesgo permitido y ese peligro se debe haber cumplido en el resultado concreto, y excepcionalmente la imputación pude desaparecer si el tipo no abarca la evitación de tales peligros ( $\mathrm{p}$. 363 - 364).

En cambio, en los tipos de peligro, se necesita la producción de un peligro real para el objeto protegido (tipo de peligro concreto) o realizar una conducta peligrosa sin tener un resultado de peligro concreto (tipo de peligro abstracto); estos tipos de peligro se diferencian del de lesión en que el primero solicita un riesgo determinado y el segundo un resultado lesivo concreto.

Se concibe de este modo que estos tipos penales, que pueden adecuar las consecuencias jurídicas de un siniestro automovilístico (muerte o lesiones), requieren un efectivo resultado que excede el peligro en el que se pueden poner dichos bienes jurídicos. Por lo que son tipos de lesión que indudablemente requieren un dolo de lesión, no se hace presente en el despliegue de quien comete homicidio o lesiones en un accidente automovilístico al ejecutarlo en todo caso con un dolo de peligro. Al no responder a la estructura típica no es posible subsumir una conducta semejante en estos delitos dolosos, debiéndose aplicar residualmente para no dejar estos casos en la impunidad una estructura culposa: la clara violación del deber de cuidado (carreras callejeras, maniobras arriesgadas, pasar cuando el semáforo no lo permite) es la determinante en el hacer delictivo.

Se debe volver sobre lo ya mencionado, lo determinante es lo volitivo: la peligrosidad abarcada por el dolo eventual, pero el resultado típico (lesiones o muerte) subsumida en la culpa consciente o con representación.

\section{La culpa temeraria como solución equitativa de política criminal}

La pena a cumplir cuando se concreta una violación del deber de cuidado grave se presenta como exigua, sobre todo cuando se está hablando de bienes jurídicos afectados en el contexto de la conducción vial. 
Es acertado recurrir al concepto de la temeridad, el cual permite que el poder punitivo del Estado recaiga sobre el sujeto activo con una mayor rigurosidad: el comportamiento imprudente temerario se presenta como inobservancia grave de las normas, por lo que el descuido no sólo no considera la realización de la situación típica, sino que también presenta un grave desprecio de la altamente posible realización del tipo.

La reforma introducida en el 2017, modificando la estructura de los tipos culposos en caso de siniestro vehicular es un avance, ya que de esta manera se obtiene un resultado más acorde al injusto cometido. La sustancia de la norma comparte los postulados defendidos, no obstante, existen supuestos ya marcados que no se condicen con un derecho penal de acto.

Salidas como la propuesta posibilitan poner a la dogmática al servicio de la comunidad, dando repuestas y siendo respetuosas de los principios constitucionales y de última ratio.

\section{CONCLUSIONES}

Una de las cuestiones más debatidas por los expertos en materia penal es la delgada y mínima diferencia entre el dolo eventual y la culpa con representación. Las líneas que precedieron no hicieron otra cosa que analizar esta problemática en una situación fáctica cotidiana: los accidentes automovilísticos.

Desde su creación, el derecho tiene como fin regular conductas humanas por medio de parámetros moralmente justos y aceptados en la sociedad. Es prudente siempre percibir cómo los institutos jurídicos son herramientas útiles para el mejor devenir de la vida humana, debiendo siempre respetar los postulados que imperan cada rama del derecho.

Situaciones como las descriptas en el presente artículo generan mucha repercusión y aflicción social, debiendo siempre procurar por la aplicación justa de las consecuencias jurídicas, pero sin desatender los principios rectores del derecho penal.

La intención es poner en objeto de debate una problemática que nos subyace a todos, ya que cualquier habitante puede ser víctima de un siniestro vehicular, debiendo ser igualmente cuidadosos en el análisis dogmático para evaluar la solución objetivamente correcta. 
Desde esta perspectiva, sostenemos que el aspecto central en estos tipos de comportamiento es la faz volitiva, siendo conteste en sostener que no es lo mismo la generación de peligro que la lesión. La primera alcanzada por un obrar riesgoso (siendo conscientes del mismo) y el segundo requiere una actitud interna que esté dirigida a un fin determinado (extremo no acaecido en los casos descriptos y explicados).

\section{REFERENCIAS}

Argibay, J. E., Damianovich, L. T., Moras, J. R. y Vergara, E. K. (1972). Derecho Penal. Parte general. Buenos Aires: Ediar.

Bacigalupo, E. (1999). Derecho Penal Parte General. Buenos Aires: Hammurabi

Cesano J.D. y Comuñez F.M. (2015). Homicidio y lesiones imprudentes en el tránsito vehicular. Buenos Aires: Hammurabi.

Garibaldi G.E. y Pitlevnik L.G. (2007). Delimitación del dolo y la culpa en el ilícito penal. Buenos Aires: Ad Hoc.

Jakobs, G. (1995). Derecho Penal, Parte General, Fundamentos y teoría de la imputación. Madrid: Marcial Pons.

Pérez, G. (2011). El dolo eventual hacia el abandono de la idea de dolo como estado mental. Buenos Aires: Hammurabi.

República Argentina. Congreso de la Nación Argentina. (22 de diciembre de 2016). Código Penal. [Ley 27.347]. Recuperado de http://servicios.infoleg.gob.ar/infolegInternet/anexos/270000-274999/270433/norma.htm

República de Argentina. Cám. Nac. de Casación Penal Sala III (2 de septiembre de 2005). Cabello, Sebastián s/recurso de casación - DO: 680/2005.

República de Argentina. CCrim $1^{\circ}$ nominación Catamarca. (5 de septiembre de 2007). Figueroa, Edgardo E.. DO: LLNOA, 2008-73.

República de Argentina. CPenal Jujuy, Sala I. (27 de marzo de 2007). Echazú, Rodolfo. DO: LLNOA, 2007-1037.

República de Argentina. TRcrim nº 6 Morón. (2 de mayo de 2006). Aldao, Cristian A.. DO: LLBA, 2006-822.

República de Argentina. Cam. Nac. Del Crim y Corr., Sala II. (5 de julio de 1977). 
República de Argentina. CRim Nº1 Trelew. (6 de marzo de 2002). Wenner, Fransisco A.. DO: LL, 2002-B 547, y Dj, 2002-1-916. República de Argentina. CGaranPen Quilmes, Sala I. (26 de junio de 2001). Soto, Diego G.. DO: LLBA, 2002-119.

República de Argentina. Capel Noroeste Chubut, Sala B. (2 de junio de 1998). Ouvrad, Pablo. DO: LL, 1998-D-821, y DJ, 1998-3-852.

República de Argentina. $3^{\circ}$ CrimCOrr. La Plata, Sala II. (14 de marzo de 1995). Cañas, Eduardo. DO: LLBA, 1995-410.

República de Argentina. Cpen Santa Fe, Sala I. (26 de noviembre de 1986). L, A.R.”. DO: RepLL, 1988-2, N³.

Rodríguez, T. (2004). Delitos de peligro, dolo e imprudencia. Buenos Aires: Rubinzal Culzoni.

Roxin, C. (2007). La Estructura de la Teoría de Delito. Derecho Penal. Parte general. Tomo 1 Fundamentos. Madrid: Civitas.

Rusconi, M. y Kierszenbaum, M. (2016). Elementos de la parte general del derecho penal. Buenos Aires: Hammurabi.

Sancinetti, M. A. (1991). Teoría del delito y disvalor de acción, una investigación sobre las consecuencias prácticas de un concepto personal de ilícito circunscripto al disvalor de la acción. Buenos Aires: Hammurabi.

Stratenwerth G. (2016). Derecho Penal. Parte General I. Buenos Aires: Hammurabi.

Tenca, M. (2010). Dolo eventual. Buenos Aires: Astrea.

Terragni, M. C. (2009). Dolo eventual y culpa consciente. Adecuación de la conducta a los respectivos tipos penales. Buenos Aires: Rubinzal Culzoni.

Vitale, G. L. (2013). Dolo eventual como construcción desigualitaria y fuera de la ley. Un supuesto de culpa grave. Buenos Aires: Editores Del Puerto.

Zaffaroni, E. R., Alagia, A. y Slokar, A. (2007). Manual de Derecho Penal. Parte general. Buenos Aires: Ediar. 